\title{
Guidelines on the Clinical Evaluation of Medicinal Products for Treatment of Dyslipidemia
}

\author{
Shizuya Yamashita ${ }^{1}$, Daisaku Masuda ${ }^{1}$, Masahiro Akishita², Hidenori Arai ${ }^{3}$, Yujiro Asada ${ }^{4}$, \\ Kazushige Dobashi ${ }^{5}$, Kensuke Egashira ${ }^{6}$, Mariko Harada-Shiba ${ }^{7}$, Kenichi Hirata ${ }^{8}$, Shun Ishibashi ${ }^{9}$, \\ Kouji Kajinami ${ }^{10}$, Makoto Kinoshita ${ }^{11}$, Koichi Kozaki ${ }^{12}$, Masafumi Kuzuya ${ }^{13}$, Masatsune Ogura ${ }^{14}$, \\ Tomonori Okamura ${ }^{15}$, Kayoko Sato ${ }^{16}$, Hitoshi Shimano ${ }^{17}$, Kazuhisa Tsukamoto ${ }^{18}$, Masayuki Yokode ${ }^{19}$, \\ Koutaro Yokote ${ }^{20}$ and Masayuki Yoshida ${ }^{21}$
}

${ }^{1}$ Rinku General Medical Center, Osaka, Japan

${ }^{2}$ Department of Geriatric Medicine, Graduate School of Medicine, the University of Tokyo, Tokyo, Japan

${ }^{3}$ National Center for Geriatrics and Gerontology, Aichi, Japan

${ }^{4}$ Department of Pathology, Faculty of Medicine, University of Miyazaki, Miyazaki, Japan

${ }^{5}$ Department of Pediatrics, Enzan Citizen Hospital, Yamanashi, Japan

${ }^{6}$ Department of Cardiovascular Medicine, Kyushu University Graduate School of Medical Sciences, Fukuoka, Japan

${ }^{7}$ Department of Molecular Pathogenesis, National Cerebral and Cardiovascular Center Research Institute, Osaka, Japan

${ }^{8}$ Division of Cardiovascular Medicine, Department of Internal Medicine, Kobe University Graduate School of Medicine, Hyogo, Japan

${ }^{9}$ Division of Endocrinology and Metabolism, Department of Medicine, Jichi Medical University, Tochigi, Japan

${ }^{10}$ Department of Cardiology, Kanazawa Medical University, Kanazawa, Japan

${ }^{11}$ Toray Industries, Inc., Tokyo, Japan

${ }^{12}$ Department of Geriatric Medicine, Kyorin University School of Medicine, Tokyo, Japan

${ }^{13}$ Department of Community Healthcare and Geriatrics, Nagoya University Graduate School of Medicine, Aich, Japan

${ }^{14}$ Department of Molecular Innovation in Lipidology, National Cerebral and Cardiovascular Center Research Institute, Osaka, Japan

${ }^{15}$ Preventive Medicine and Public Health, Keio University School of Medicine, Tokyo, Japan

${ }^{16}$ Department of Cardiology, Tokyo Women's Medical University, Tokyo, Japan

${ }^{17}$ Department of Internal Medicine (Endocrinology and Metabolism), Faculty of Medicine, University of Tsukuba, Ibaraki, Japan

${ }^{18}$ Department of Internal Medicine, Teikyo University School of Medicine, Tokyo, Japan

${ }^{19}$ Department of Clinical Innovative Medicine, Kyoto University Graduate School of Medicine, Kyoto, Japan

${ }^{20}$ Department of Endocrinology, Hematology and Gerontology, Chiba University Graduate School of Medicine, Chiba, Japan

${ }^{21}$ Division of Medical Genetics, Medical Hospital of Tokyo Medical and Dental University, Tokyo, Japan

\section{Key words: Guidelines, Clinical Evaluation, Medicinal Products, Treatment of Dyslipidemia, LDL-C, Japanese}

\section{Introduction}

In 1983, the Ministry of Health, Labor and Welfare of Japan has organized a "Special Research Team for Primary Hyperlipidemia" with Prof. Sei-ichiro Tarui of Osaka University as a chairperson, in order to start a nationwide investigative research on primary hyperlipidemia. Leading the efforts at basic/clinical studies, the team established diagnostic criteria on various symptoms of primary hyperlipidemia seen among Japanese people and informed the public, both domes- tic and international, of the importance of hyperlipidemia treatment. Based on their findings, the Ministry compiled a "Guideline for Clinical Evaluation Methodology for Antihyperlipidemic Drugs (January 05, 1988, Pharmaceutical Notification No. 1-1)." This guideline presented the general procedures for the planning, implementation, evaluation, and other specifics of clinical studies in order to evaluate the clinical efficacy of novel (oral) antihyperlipidemic drugs.

Hyperlipidemia has been identified to cause several health disorders such as acute pancreatitis in

Address for correspondence: Shizuya Yamashita, Department of Cardiology, Rinku General Medical Center, Izumisano, Osaka 598-8577, Japan

E-mail: s-yamashita@rgmc.izumisano.osaka.jp

Received: July 6, 2020 Accepted for publication: July 10, 2020

Copyright@2020 Japan Atherosclerosis Society

This article is distributed under the terms of the latest version of CC BY-NC-SA defined by the Creative Commons Attribution License. 
patients with marked hypertriglyceridemia and xanthoma (tendon xanthoma and skin xanthoma) in patients with hypercholesterolemia and/or hypertriglyceridemia. Furthermore, hyperlipidemia is regarded as the most important risk factor for atherosclerosis that forms a basis for cerebro-cardiovascular diseases, which are considered as one of the major causes of death in Japan along with cancer. Though administration of antihyperlipidemic drugs has long been focused on preventing the onset and progression of atherosclerotic diseases, evaluating the prophylactic effects of these agents remains to be difficult as there is no evidence available to support those effects during the developmental stage of drugs. Therefore, the therapeutic efficacy of these agents was limited to hypercholesterolemia in the previous guideline.

In the meantime, the "Japan Atherosclerosis Society (JAS) Guideline for Diagnosis and Treatment of Hyperlipidemia" was first published by the JAS in 1997 for clinical practitioners; since then, the guideline has been revised at 5-year intervals. Meanwhile, the "Guidelines for Preventing Atherosclerotic Diseases," which considers risk factors based on the evidence available in Japan, was published in 2002.

In the 2007 edition of the Guidelines for Preventing Atherosclerotic Diseases, "hyperlipidemia" was rephrased as "dyslipidemia," and LDL-C was targeted as the major risk factor instead of total cholesterol. To date, the 2017 edition of the Guidelines for Preventing Atherosclerotic Diseases is the latest guideline for clinical practice published by the JAS, in which absolute risks are assessed using the Suita score. Meanwhile, development and commercialization have continued for novel drugs, such as PCSK9 inhibitors and MTP inhibitors, and a selective $\operatorname{PPAR} \alpha$ modulator for dyslipidemia.

As described above, even with the availability of newly developed therapeutic modalities for dyslipidemia, a great number of patients are still determined to suffer from refractory cases of dyslipidemia, especially primary hyperlipidemia. With both Japanese and overseas researchers being involved, more and more drugs have been developed using new approaches, such as low-molecular compounds, nucleic acid medicine, antibody medicine, genetic treatment, and cellular treatment. In June 2016, the European Medicines Agency (EMA) was able to publish a guideline in developing new antilipidemic drugs titled the "Guideline on Clinical Investigation of Medicinal Products in the Treatment of Lipid Disorders." Thus, we would like to formulate a new guideline on antilipidemic drug development to echo the activity of EMA in view of achieving worldwide harmonization.
Amid these circumstances, the JAS has been in communication with the Pharmaceuticals and Medical Devices Agency in Japan, where they organized a research team chaired by Prof. Shizuya Yamashita, the president of the JAS, under the auspices of the Special Scientific Research Project of the Ministry of Health, Labor and Welfare "Research for the Streamlining and International Standardization of Antilipidemic Drug Clinical Evaluation."

\section{Target Scope and Populations}

\subsection{Target Scope of this Guideline}

This guideline is applicable to clinical evaluation of antilipidemic drugs, including serum LDL-C-lowering drugs, serum TG-lowering drugs, and anti-hypoHDL-C-emic drugs.

The indications include (1) hypercholesterolemia (with LDL-C values being evaluation criteria), (2) hypertriglyceridemia, (3) hypo-HDL-cholesterolemia, and (4) familial hyperlipidemia (familial hypercholesterolemia, familial combined hyperlipidemia, familial type III hyperlipidemia, and primary hyperlipidemic conditions including primary hyperchylomicronemia). This guideline covers antilipidemic drugs clinically developed in order to attenuate the progression of atherosclerosis, suppress cerebro-cardiovascular events, and further prevent the onset of pancreatitis.

Additionally, drugs with novel action mechanisms and medicinal effects need to demonstrate their proven efficacy in suppressing cerebro-cardiovascular events according to the type of the indicated dyslipidemia. Moreover, both short-term and long-term adverse reactions need to be taken into consideration in terms of the characteristics of each drug.

\subsection{Target Population of Clinical Studies}

In screening subjects for the clinical evaluation of antilipidemic drugs, it is essential to select a population that is appropriate for the target indications/ effects. For the diagnostic criteria of dyslipidemic conditions, including hyperlipemia (evaluated as hyperLDL-cholesterolemia), hypertriglyceridemia, and hypo-HDL-cholesterolemia, refer to the relevant clinical guidelines for the corresponding region (for Japan, the latest version (the 2017 edition as of March 2020) of the Japan Atherosclerosis Society Guidelines for Prevention of Atherosclerotic Cardiovascular Diseases 2017 published by the JAS). Likewise, for the diagnosis of familial hyperlipidemia, refer to the latest classification of primary hyperlipidemia as published by the Research Committee on Primary Hyperlipidemia designated by the Japanese Ministry of Health, Labor and Welfare. 
In some cases of familial hyperlipidemia, patients may require higher doses of administration than usual. Accordingly, when setting proper drug doses and levels for patients with familial hyperlipidemia, it may be necessary to consider performing separate tests involving only familial hyperlipidemia patients while excluding patients with non-familial hypercholesteremia (hyper-LDL-cholesterolemia).

Recent reports have demonstrated that pharmacotherapy is also an effective method in treating dyslipidemia in patients aged 75 years or older. Therefore, future pivotal clinical studies will need to include a sufficient number of patients, in order to possibly evaluate its drug efficacy and safety for a population aged 75 years or older. For the evaluation of patients aged 75 years or older, also refer to the "Studies in support of special populations: geriatrics" (ICH E7 https://www.pmda.go.jp/int-activities/int-harmony/ ich/0029.html).

Patients with dyslipidemia are found to likely have complications such as hypertension, diabetes mellitus, chronic kidney disease (CKD), and cerebrocardiovascular diseases. Drug efficacy and safety for patients with these complications should be evaluated as necessary using subgroup analyses of clinical studies.

\section{Evaluation of Anti-Dyslipidemic Drugs}

In evaluating anti-dyslipidemic drugs, several evaluation factors affecting the clinical outcome can be employed, besides the changes in lipid levels.

\subsection{Efficacy Evaluation Items \\ 3.1.1. Cerebro-Cardiovascular Events and Mortality}

One major objective in treating dyslipidemia is to suppress cerebro-cardiovascular events and prevent deaths. Here the term "cerebro-cardiovascular event" refers to a combination of four episodes: (1) cardiovascular death, (2) myocardial infarction, (3) coronary artery revascularization, and (4) stroke. Given these therapeutic goals, efficacy evaluation of anti-dyslipidemic drugs should basically demonstrate improvement in total mortality. In fact, the results of many previous clinical studies have indicated that HMG-CoA reductase inhibitor (statin) can suppress cerebro-cardiovascular events and reduce total mortality. Consequently, in order to evaluate the efficacy of novel anti-dyslipidemic drugs, observing improvements in LDL-C levels is considered to be an effective index. For drugs that improve lipid parameters other than LDL-C, it is favorable to demonstrate the suppression of the occurrence of cerebro-cardiovascular events and mortality before approval; otherwise, the relevant regulatory authorities should be consulted based on scientific findings such as image diagnosis.

\subsubsection{Lipid Values (LDL-C, HDL-C, TG, and Non- $H D L-C)$}

Reduced LDL-C levels from baseline after antidyslipidemic drug administration can be used as a major efficacy evaluation item for patients with hypercholesterolemia. Regarding measurement of $\mathrm{TG}$ or HDL-C levels, data demonstrating their clinical utility compared with LDL-C remain to be lacking. Therefore, when no scientific evidence for clinical efficacy is shown at the time of application, changes in TG and HDL-C should be evaluated as a secondary efficacy index, as opposed to the indices for evaluating other major medications. When LDL-C is hard to evaluate due to high TG levels in postprandial blood testing or similar situations, non-HDL-C can also be utilized as an evaluation item.

\subsubsection{Vascular Damage (Target Organ Damage)}

Important target organs that incur damage from dyslipidemia are the heart, brain, kidneys, and arteries. In the event of such target organ damage, atherosclerosis-derived calcification may occur on the basis of vascular dysfunction. The image diagnosis methods hitherto established for vascular dysfunction are intima-media thickness (IMT) measurement of carotid arteries by ultrasound,computed tomography (CT), magnetic resonance imaging (MRI), coronary artery contrast radiography, intravascular ultrasound (IVUS), and optical coherence tomography (OCT). Of these measurements, applicable evaluation items may include changes in atherosclerotic plaque levels before and after drug administration by IMT and IVUS, remodeling by CT, and morphological evaluation of coronary artery plaques.

\subsubsection{Acute Pancreatitis}

TG has been shown to correlate with acute pancreatitis ${ }^{1)}$. In cases of severe hypertriglyceridemia, improving TG levels is considered to be an evaluation item.

\subsection{Efficacy Evaluation Methods}

\subsubsection{Evaluating the Incidence and Mortality of Cerebro-Cardiovascular Events}

In order to evaluate the incidence and mortality of cerebro-cardiovascular events in a clinical study on anti-dyslipidemic drugs, an evaluation committee should be established, independent of the researchers of the clinical study. The evaluation committee will be tasked to evaluate the efficacy of the test drug in relation to the incidence and mortality of cerebro-cardio- 
vascular events and assess each case in accordance with the set evaluation items.

Unlike more objective events such as death, myocardial infarction, and stroke (hard endpoints), events more liable to subjective interpretations such as angina pectoris, transient cerebral ischemia, heart failure, and PCI/CABG (soft endpoints) need to have diagnostic criteria specifically defined in the implementation protocol in advance before they are used as evaluation items. When an event is reported, the evaluation committee should ascertain whether that event satisfies the defined diagnostic criteria.

\subsubsection{Evaluation of Lipid Levels}

In principle, fasting blood lipid levels are measured using blood samples collected following at least 10 hours of fasting. LDL-C levels are calculated using the Friedewald equation, with the direct and BQ methods also used optionally. Non-HDL-C level signifies a sum of cholesterol contained in atherogenic lipoproteins other than HDL; it includes cholesterol contained in LDL, remnant lipoproteins, and Lp(a). For a population with high non-HDL-C levels, it is preferable to not only measure LDL-C and TG levels but to also evaluate specific lipoproteins, including remnant lipoproteins, small dense LDL (sdLDL), and $\operatorname{Lp}(\mathrm{a})$.

These lipid values are often evaluated based on either change rate or change level before and after test drug administration. Before collecting pre-administration blood samples, it is necessary to wash out drugs that may affect the efficacy evaluation of the test drug for an appropriate period of time. For a test drug targeting lipid values other than LDL-C (e.g., HDL-C), the evaluation items as mentioned in Section 3.1.1 should be included. The achievement rate of the management goal for each category after stratifying subjects depending on the risk factors may also be used as an evaluation item.

\subsubsection{Evaluation of Vascular Damage (Target Organ Damage)}

Vascular damage in the heart, brain, kidneys, and large arteries is determined to be correlated with an increase in the incidence and mortality of cerebro-cardiovascular diseases. Image diagnostic technology is found to be critical as an index for atherosclerosis progression in the target organ. The image diagnostic methods that have been established for the quantitative evaluation of atherosclerosis, used for drug efficacy evaluation, and demonstrated to correlate with cerebro-cardiovascular accidents are as follows: IMT, quantitative coronary artery angiography, and coronary artery IVUS. Additionally, the following evalua- tion items have been increasingly utilized: plaque levels, histological characteristic evaluation, multidetector-row computed tomography, maximum intensity projection, coronary artery calcification, multiplanar reconstruction (MPR) evaluation of the stenosis level and plaque characteristics, and OCT and MRI evaluation of vascular shape. The data on their quantitativeness has been accumulating. Various indices used in these measurement systems may possibly serve as valid evidence as markers of atherosclerosis progression in the stages of drug development, including the dose/ indication setting stage.

\section{Selection of Patients (Age/Sex/Complication)}

In general, a test population used to evaluate the efficacy or safety of a novel anti-dyslipidemic drug is selected based on the type of dyslipidemia for which the said drug is indicated. Additionally, a test population should include both male and female patients at a proper ratio. Regarding infant patients, another chapter will be dedicated for its discussion. In terms of evaluating both the efficacy and safety of anti-dyslipidemic drugs in older patients, a test population should include a sufficient number of subjects aged 75 years or older.

Moreover, in order to properly evaluate the clinical outcome, it is preferable to have the risk level within a test population be homogenized or the intrapopulation risk be stratified. This makes it easier to interpret the test results. To perform the subpopulation analysis consistent with the analysis of the entire test population, pivotal studies need to include a certain number of high-risk patients with conditions such as coronary artery disease, type 2 diabetes, CKD, and non-cardiogenic cerebral infarction.

Furthermore, to evaluate drug efficacy for patients with familial hypercholesterolemia (heterozygotes and homozygotes), which is an atypical form of dyslipidemia, it is preferable to consider performing a separate clinical study while taking into account cholesterol levels and genetic traits.

\section{Methods and Designs of Clinical Studies}

In all clinical studies, therapies for improving lifestyle habits (diet therapy, exercise training, etc.), as described in guidelines and other documents, should be introduced prior to anti-dyslipidemic drug interventions. Establishing enrollment criteria for clinical studies is important to ensure that the diagnostic procedures for the relevant disease are reliable. For patients who underwent treatment for other types of 
dyslipidemia, setting a sufficient washout period should be considered prior to the single regimen of the target anti-dyslipidemic drug. It should also be noted that lifestyle habits (drinking alcohol, smoking, and exercise) and consumption of nutraceutical foods and supplements remained unchanged throughout the test period.

\subsection{Pharmacodynamics}

A pharmacodynamic study must include the evaluation of action mechanisms, tolerability, duration of action, and relevant clinical and hemodynamic parameters. For instance, measurements of various clinical test values may sometimes be necessary to examine blood pressure, hepatic function, renal function, immune reaction, and off-target effects such as complement activation. If such off-target effects are pointed out at an early stage of development, special care is required during the ensuing exploratory or confirmative clinical trials.

\subsection{Pharmacokinetics}

Special attention needs to be paid for the pharmacokinetic interaction between the test drug and other drugs that may possibly concomitantly used in the study population ${ }^{2,3)}$. In some cases, tests targeting subgroups will be necessary in order to evaluate the changes in pharmacokinetics stemming from dietary contents, environmental factors like smoking, and genetic polymorphisms. Depending on the dynamic feature of the pharmaceutical product, testing patients with renal and hepatic dysfunction should be considered. Care is also required for an excessive increase in blood concentrations, delayed metabolism, and tissue accumulation. It is preferred to fully evaluate drug interactions and pharmacokinetic profiles.

\subsection{Clinical Trial}

\subsubsection{Dose-Finding Exploratory Trial}

Dose-response tests should be performed in a randomized, placebo-controlled, double-blind fashion. To establish an effective dose range and determine an optimum dose, at least three different doses should be tried for evaluation. As a primary test design, a parallel-group comparison design that allocates to fixed doses is employed. The effects on lipid values should be examined independently by dose. Additionally, it is a must to clearly predefine administration schedules. Administration periods should range from 4 weeks to approximately 3 months (longer when necessary) and should be determined based on the study objectives and drug characteristics.

\subsubsection{Confirmatory Trial}

\subsubsection{Evaluation of Anti-Dyslipidemic Effects as A Monotherapy}

For drugs targeting LDL-C, placebo-controlled testing by monotherapy is not recommended given the efficacy and safety of statin, except for clinical studies specifically involving statin-intolerant ${ }^{4)}$ patients with dyslipidemia. When targeting other lipid values, placebo-controlled tests can also be considered; however, benefits of the drug should also be determined and evaluated in addition to its anti-dyslipidemic effects. Meanwhile, tests targeting statin-intolerant patients with dyslipidemia should be separately examined, or they should be tested as a predesignated alternate therapy group within the clinical study.

To evaluate the efficacy and safety of novel antidyslipidemic drugs, comparing them with already established therapeutic modalities is preferable. To select appropriate control drugs, pharmacological classifications and the type/indication of the antilipidemic drug should be considered. For comparison within the same pharmacological classification, attention should be paid to the administered dose of each drug based on its relative titer. In an anti-dyslipidemic drug trial, the dose and administration schedule should be predetermined based on the dose-finding test for the target population. If the evaluation of lipid values is set as the primary objective, the test period should last at least 3 months (for known action mechanisms) and preferably 12 months (for others). When gradually increasing the dose, the study protocol should be observed. Precautionary measures, such as changing the dose after a certain period suitable for evaluation, are required.

\subsubsection{Evaluation of Anti-Dyslipidemic Effects When Used Concomitantly with Other Anti-Dys- lipidemic Drugs}

When a novel drug is used concomitantly with other already available drugs, the clinical study should involve patients unable to achieve full control with known drugs. Specifically, to evaluate the LDL-Clowering effect of the newly added secondary anti-dyslipidemic drug targeting hyper-LDL-cholesterolemia, the maximum tolerated dose of statin should be administered to each individual patient as designated by the indications/dosage of the approved pharmaceutical products, except for statin-intolerant patients. In general, it is difficult to acknowledge combination therapies as first-line therapies based solely on their effects against LDL-C, TG, HDL-C, and non-HDLC. Therefore, additional data on decreased cerebrocardiovascular events or decreased mortality will be required. 


\subsubsection{Effectiveness Evaluation in Clinical Out- comes}

To demonstrate its effectiveness in clinical outcomes (especially cardiovascular diseases), it is preferable to perform a double-blind clinical study with longterm parallel-group comparison. In this context, clinical studies include international joint studies that involve a sufficient number of Japanese patients. Both superiority and non-inferiority studies are applicable. If there is no established cure for the target population, a placebo-controlled study may be tolerated.

\section{Safety}

Anti-dyslipidemic drugs require comprehensive safety evaluation as they are used for long-term administration. A preclinical study for detecting/evaluating potential adverse events should be planned during the early stages of drug development (preferably by the time of implementing an exploratory clinical study). At that time, it is necessary to factor in pharmacological safety and consider major toxicological findings. In conventional anti-dyslipidemic drugs, a warning was issued specifically for muscular disorders including rhabdomyolysis. Accordingly, an increase in the risk of a specific adverse event is a serious concern, so much so it may necessitate a long-term safety trial before and after approval.

Sometimes, it may be difficult to determine the safety of pharmaceutical products by only using the long-term safety trial implemented prior to approval. For instance, statin reportedly increased the new onset of diabetes mellitus in a meta-analysis performed in a cerebro-cardiovascular event-suppression study using statin $^{1)}$. Meanwhile, other reports have indicated that the extent of increase has varied depending on the type of drug. Therefore, given the properties and classification of the test drug, information on long-term safety risk should be gathered and evaluated as necessary as part of the post-marketing collection of safety data.

\subsection{Safety for Target Organs}

To ensure the safety of individual organs, the results of the non-clinical and clinical trials based on the action mechanism of the applicable compound should reflect the safety information confirmed in other compounds. Particular caution is required for the following organs:

Liver: Hepatic functions should be measured and evaluated on a regular basis. Safety evaluation should be performed appropriately so that the information of patients with varying degrees of hepatic dysfunction (Child-Pugh classification) is reflected properly in the package insert or other documentation.

Kidney: Non-clinical studies have reported renal toxicity in some anti-dyslipidemic drugs. In patients with renal dysfunction using some anti-dyslipidemic drugs, muscle-related adverse events may be aggravated. Therefore, careful consideration is required in the course of development.

Muscle: Various anti-dyslipidemic drugs, especially statin, have reportedly induced an increase in creatine kinase (CK) accompanied by muscle pain symptoms. In a clinical trial intended for drug development, it is recommended that CK levels be checked on a regular basis after the onset of muscle symptoms as part of safety evaluation. In the event of serious muscular disorder, which is usually not very frequent, post-marketing surveys and the like should be planned.

\subsection{Safety for Cerebro-Cardiovascular Events}

Clinical and non-clinical trials implemented during the development of anti-dyslipidemic drugs make it possible to evaluate the safety of the test drug for cerebro-cardiovascular events (including cerebral hemorrhage). These trials are expected to include items capable of properly evaluating cerebro-cardiovascular risk profiles. To ascertain whether safety profiles are established for products with novel action mechanisms or cerebro-cardiovascular events, products belonging to drug classes that have been suggested to possibly increase certain cerebro-cardiovascular events should be scrutinized. Cerebro-cardiovascular risk evaluation should be conducted in clinical trials implemented for approval application or post-marketing information collection.

\subsection{Concomitant Drugs}

Active safety evaluation should be conducted for concomitant use with other anti-dyslipidemic drugs, especially for conditions that are frequently accompanied by dyslipidemia. During long-term administration, it is critical to carefully scrutinize the safety of concomitant use with frequently used adjuvants that are expected to interact with the developed drug (e.g., sharing drug-metabolizing systems, forming a substrate for the same transporter) through post-marketing surveys and the like.

\subsection{Drugs with Novel Action Mechanisms}

As pharmaceutical products target specific molecules for the purpose of treating dyslipidemia, efforts are underway to develop monoclonal antibodies and nucleic acid medicines (antisense oligonucleotides, small interfering RNA (siRNA), etc.). The majority of these drugs are medium to high molecular compounds 
and are administered via injection. Accordingly, each individual drug may have diverse characteristics in terms of immune reaction, length of half-life, and expression of molecules relating to the target protein. Therefore, in accordance with the "Guideline on how to proceed with the formulation of study implementation protocols including post-marketing surveys etc. for pharmaceutical products" (Pharmacopoeia Notification No. 0314-4; Pharmaceutical Safety Notification NO. 0314-4, March 14, 2019), the efficacy and safety of these drugs should be verified based on the post-marketing information collection plan established to achieve its objectives.

Furthermore, pharmaceutical products with novel action mechanisms may have clearly different pharmacological actions and safety profiles than the existing products. During development, the target population for administration should be carefully selected based on the accumulated data on efficacy and safety (e.g., targeting patients that are expected to benefit the most from the developed product).

\section{Special Populations}

While planning a clinical trial for anti-dyslipidemic drugs, researchers may need to consider changing the dose or administration schedule for the following special populations compared with the more common populations.

\subsection{Older Patients}

Compared with the younger populations, older patients are more susceptible to possible threats to quality of life due to conditions like sarcopenia, frailty, and malnutrition. The goals of any dyslipidemia treatment for older patients should be to prevent cerebrocardiovascular diseases and ultimately extend healthy life expectancy ${ }^{5)}$.

To fully evaluate drug efficacy and safety for older patients, a clinical trial should be designed to incorporate a sizable number of older patients, especially those aged 75 years or older. As many older people have impaired renal functions, attention should be paid to pharmacokinetics. Older people often have multiple preexisting conditions and are liable to polypharmacy; therefore, further information is needed on the impact of concomitant drugs. Additionally, measures need to be taken for older people who tend to have low adherence to the dosage regimen. Preferably, the researcher should be able to track their administration status in the actual clinical setting.

\subsection{Patients with Organ Damage}

For the enrollment of patients with organ dam- age, risks should be fully considered based on the action mechanism and major metabolic pathways. Moreover, the results of the non-clinical and clinical studies should be verified to confirm the safety of other compounds. Particular caution is required for the following organs:

Liver: One of the adverse reactions of anti-dyslipidemic drugs is hepatic dysfunction; hence, hepatic functions should be tested on a regular basis. This information on the patient's hepatic condition (diagnosis, degree of hepatic dysfunction, etc.) should be included in the report.

Kidney: One of the adverse reactions of anti-dyslipidemic drugs is renal dysfunction; hence, renal functions should be tested on a regular basis. Moreover, patients with renal dysfunction are known to be more predisposed to muscle-related adverse events caused by anti-dyslipidemic drugs. For the definition of the degrees of renal dysfunction, ambiguous expressions (e.g., mild, moderate, and serious) should be avoided; instead, seriousness should be represented by levels of estimated glomerular filtration rate (eGFR) or creatinine clearance.

Past history of muscle disorder: Anti-dyslipidemic drugs, including statin, may cause some symptoms accompanied by an increase in CK. Therefore, it is essential to check for myopathy and measure blood CK concentrations on a regular basis during the development programs, clinical trials, and post-marketing surveys.

Past history of muscle disorder: Hypocholesterolemia has been reported to increase the pathogenic risk of hemorrhagic stroke ${ }^{6}$. Therefore, caution is needed when enrolling people with a past history of cerebral hemorrhage.

As part of the data on the test product, information relating to subjects with organ damage should be recorded along with the metabolic characteristics and loss pathways of the test product.

\subsection{Children}

The efficacy and safety of antilipidemic drugs for children (age 15 years or younger) are yet to be fully elucidated. Moreover, it should be noted that children in their growth stage have different follow-up items than adults. Before use, full explanation should be provided to the guardian of the patient, and the guardian's consent should be obtained. For considerations during clinical evaluation, refer to the "Clinical Evaluation Considerations for Children Evaluated along with Adults" (Public comment recruitment from January 16 to February 15, 2019). Additionally, the Japan Pediatric Society has also been promoting novel drug development in the field of infantile care ${ }^{7)}$; 
hence, there publications should be noted.

For the criteria and treatment of pediatric dyslipidemia, consult with the latest guidelines of the respective countries. As of March 2020, pharmacotherapy for pediatric dyslipidemia is only recommended, in principle, for Familial Hypercholesterolemia $(\mathrm{FH})$ patients aged 10 or older who maintain high lipid levels after other therapies, including dietary guidance. They require different drugs and control standards than adult FH patients (aged 15 years or older $)^{8)}$.

\subsection{Women}

As necessary, data on how anti-dyslipidemic drugs affect pregnancy, unborn baby, and postmenopausal biological changes should be examined. Also, sexual differences in pharmacokinetics should also be considered based on the characteristics of the pharmacological product.

\section{Legal Bases and Relevant Guidelines}

This guideline is an edited version of the 2005 edition "Guideline on Clinical Evaluation of Antilipidemic Drugs" of the "Guideline on Clinical Evaluation of Antihyperlipidemic Drugs" (Pharmacopoeia Notification No. 1-1, January 1988), which has been used as a standard for the clinical evaluation of antilipidemic drugs in Japan.

For this edition, the "Guideline on Clinical Investigation of Medicinal Products in the Treatment of Lipid Disorders" created by the EMA was referenced, and discussions within the EMA were then noted.

To enforce this guideline, relevant guidelines and notifications by the International Conference on Harmonization of Technical Requirements for Registration of Pharmaceuticals for Human Use $(\mathrm{ICH})^{9)}$ should be studied.

\section{Acknowledgements}

The authors thank Drs. Kristina Dunder, Heidi Janssen, Anna Baczynska, and Pieter de Graeff of European Medicines Agency (EMA) and Dr. Alberico L Catapano for their valuable comments on this guideline.

\section{Sources of Funding}

This work was supported by a grant from the Ministry of Health, Labor and Welfare of Japan.

\section{Conflict of Interest}

Board member: Liid Pharma. Stock holding: Liid Pharma. Honoraria: Amgen, Amgen Astellas BioPharma K.K., Astellas, Astra-Zeneca, Bayer Yakuhin, Bayer Yakuhin, Ltd., Daiichi Sankyo, DainipponSumitomo, Eisai Co., Ltd., Jansen, Kowa Company, Ltd., Kowa Pharmaceutical Co. Ltd., Kyowa-kirin, Mitsubishi-Tanabe Pharma, MSD K.K., Nippon EliLilly, Novartis, Novonordisk, Ono Phamaceutical Co,. Ltd., Otuka Pharmaceutical Factory., Inc., Pfizer, Sanofi K.K., Sumitomo Dainippon Pharma, Taisho, Takeda Pharmaceutical Company Limited., Tsumura \& Co., UCB Japan. Fees for promotional materials: Daiichi Sankyo. Total clinical research funding: Actelion Pharmaceuticals Japan Ltd., Astellas, Astellas Pharma Inc., AstraZeneca K.K., Bayer Yakuhin, Ltd., Daiichi Sankyo Company, Limited, Eisai, Eli Lilly Japan, GlaxoSmithKline plc, Hayashibara Co., Ltd., Hitachi Chemical Diagnostics Systems Co., Ltd., Kissei Pharmaceutical Co., Ltd, Kowa Company, Ltd., Kracie Pharma, Mitsubishi Tanabe Pharma Corporation, Mochida Pharmaceutical Co., Ltd, MSD K.K., Nippon Boehringer Ingelheim Co., Ltd., Ono Pharmaceutical Co., Ltd., Otsuka Pharmaceutical Co., Ltd., Rohto Pharmaceutical Co., Ltd, Sanwa Kagaku Kenkyusho Co.,Ltd., Shionogi\&Co., Ltd, SYSMEX CORPORATION, Taisho, Takeda Pharmaceutical Company Limited,, Terumo Corporation. Total scholarship grants: Abbott Vascular Japan CO.,Ltd., Actelion Pharmaceuticals Japan Ltd., Aegerion, Astellas Pharma Inc., Bayer HealthCare, Bayer Yakuhin, Ltd., BIOTRONIK Japan, Inc., Boehringer Ingelheim, Boston Scientific Corporation, Chugai Pharmaceutical, Daiichi Sankyo Co., Ltd., Dainippon-Sumitomo, Eizai Co. Ltd, FUJIFILM Holdings Corporation, Japan Eli-Lilly, Kaneka Medics, Kowa Company Limited(KOWA PHARMACEUTICAL COMPANY LTD), Mitsubishi Tanabe Pharma Corporation, MSD K.K., Nihon Medi-Physics Co., Ltd, Nippon Boehringer Ingelheim Co., Ltd., NIPPON SHINYAKU CO., LTD., Novartis Pharma K.K., Novonordisk, Ono Pharmaceutical Co., Ltd., Otsuka Pharmaceutical Co., Ltd., Pfizer Inc., Recordati, Sanofi K.K., Shionogi, Sumitomo Dainippon Pharma Co., Ltd., Taisho, Takeda Pharmaceutical Company Limited, Teijin Pharma, Tsumura. Courses endowed by companies: Abbott Medical Japan LLC. (ST. JUDE MEDICAL IS NOW ABOTT), Izumisano City, Kaizuka City, Medtronic Japan Co., Ltd., MSD, SYSMEX CORPORATION. 


\section{References}

1) Kinoshita $M$, Yokote $K$, Arai $H$, Iida $M$, Ishigaki $Y$, Ishibashi S, Seiji Umemoto S, Egusa G, Ohmura H, Okamura T, Kihara S, Koba S, Saito I, Shoji T, Daida H, Tsukamoto K, Deguchi J, Dohi S, Dobashi K, Hamaguchi H, Hara M, Hiro T, Biro S, Fujioka Y, Maruyama C, Miyamoto Y, Murakami Y, Yokode M, Yoshida H, Rakugi H, Wakatsuki A, Yamashita S, Committee for Epidemiology and Clinical Management of Atherosclerosis: Japan Atherosclerosis Society (JAS) Guidelines for Prevention of Atherosclerotic Cardiovascular Diseases 2017. J Atheroscler Thromb, 2018; 25: 846-984

2) Notification No.796 issued by Pharmaceutical and Medical Safety Bureau, Ministry of Health and Welfare. 2001. (in Japanese)

3) Guideline on drug interaction for drug development and appropriate provision of information. 2018. (in Japanese, MHLW, https://www.pmda.go.jp/rs-std-jp/standardsdevelopment/guidance-guideline/0001.html)

4) Kajinami K, Tsukamoto K, Koba S, Inoue I, Yamakawa M, Suzuki S, Hamano T, Saito H, Saito Y, Masuda S, Nakayama T, Okamura T, Yamashita S, Kagawa T, Kaneyama J, Kuriyama A, Tanaka R, Hirata A, Statin Intolerance Clinical Guide Working Group; The Japan Society of Hepatology, Japanese Society of Neurology, Japan Atherosclerosis Society, The Japanese Society for the Study of Xenobiotics: Statin intolerance clinical guide 2018. J Atheroscler Thromb, 2020; 27: 375-396

5) Satake S, Arai H: Chapter 1 Frailty: Definition, diagnosis, epidemiology. Geriatr Gerontol Int, 2020 Feb; 20 Suppl 1:7-13

6) Amarenco P, Bogousslavsky J, Callahan A 3rd, Goldstein LB, Hennerici M, Rudolph AE, Sillesen H, Simunovic L, Szarek M, Welch KMA, Zivin JA, Stroke Prevention by Aggressive Reduction in Cholesterol Levels (SPARCL) Investigators: High-dose atorvastatin after stroke or transient ischemic attack. N Engl J Med, 2006; 355: 549-559

7) Japan Pediatric Society Website (https://www.jpeds.or.jp/ modules/activity/index.php?content_id=247) (in Japanese)

8) Harada-Shiba M, Ohta T, Ohtake A, Ogura M, Dobashi K, Nohara A, Yamashita S, Yokote K, Joint Working Group by Japan Pediatric Society and Japan Atherosclerosis Society for Making Guidance of Pediatric Familial Hypercholesterolemia: Guidance for Pediatric Familial Hypercholesterolemia 2017. J Atheroscler Thromb, 2018; 25: $539-553$

9) The International Conference on Harmonization of Technical Requirements for Registration of Pharmaceuticals for Human Use (ICH) guidelines.
ICH-E1 The Extent of Population Exposure to Assess Clinical Safety for Drugs Intended for Long-Term Treatment of Non-Life-Threatening Conditions

ICH-E4 Dose-Response Information to Support Drug Registration

ICH-E5 Ethnic Factors in the Acceptability of Foreign Clinical Data

ICH-E7 Studies in Support of Special Populations: Geriatrics

ICH-E8 General Considerations for Clinical Trials

ICH-E9 Statistical Principles for Clinical Trials

ICH-E10 Choice of Control Group and Related Issues in Clinical Trials

ICH-E11 Clinical Investigation of Medicinal Products in the Pediatric Population

ICH-E14 The Clinical Evaluation of QT/QTc Interval Prolongation and Proarrhythmic Potential for Non-Antiarrhythmic Drugs

ICH-E17 General Principles for Planning and Design of Multi-Regional Clinical Trials 